\title{
The role of soluble programmed death protein-1 (sPD-1) and soluble programmed death ligand-1 (sPD-L1) in rat corneal transplantation rejection
}

\author{
Guoguo Yi ${ }^{1, A-F}$, Ruiwen $\mathrm{Yi}^{1}{ }^{1, A-D}$, Xinglu Chen ${ }^{2, B, C}$, Ling Peng ${ }^{3, A-C}$, Guogiang Huang ${ }^{4, B, C}$, Min Fu ${ }^{1, A, D}$, Xiao-he Lu ${ }^{1, E, F}$, Hongwei Li ${ }^{5, E, F}$ \\ ${ }^{1}$ Zhujiang Hospital of Southern Medical University, Guangzhou, China \\ $27^{\text {st }}$ Affiliated Hospital of Guangdong Pharmaceutical University, Kanton, China \\ ${ }^{3}$ Women \& Children's Health Institute of Futian, Shenzen, China \\ ${ }^{4}$ Meizhou People's Hospital, China \\ ${ }^{5}$ School of Biotechnology, Southern Medical University, Kanton, China \\ A - research concept and design; B - collection and/or assembly of data; C - data analysis and interpretation; \\ $D$ - writing the article; $E$ - critical revision of the article; $F$ - final approval of the article
}

\section{Xiao-he Lu \\ E-mail:luxh63@163.com \\ Funding sources \\ None declared \\ Conflict of interest \\ None declared}

Address for correspondence

Received on December 13, 2017

Reviewed on December 20, 2017

Accepted on April 9, 2018

Published online on January 13, 2021

Cite as

Guoguo Y, Ruiwen Y, Xinglu C, et al. The role of soluble programmed death protein-1 (sPD-1) and soluble programmed death ligand-1 (SPD-L1) in rat corneal transplantation rejection. Adv Clin Exp Med. 2021:30(1):93-100. doi:10.17219/acem/89803

DOI

10.17219/acem/89803

\section{Copyright}

Copyright by Author(s)

This is an article distributed under the terms of the

Creative Commons Attribution Non-Commercial License

(http://creativecommons.org/licenses/by-nc-nd/4.0/)

\section{Abstract}

Background. Immunological rejection is one of the problems in corneal transplantation. Recently, some research found out that soluble programmed death protein-1 (SPD-1) and soluble programmed death ligand protein-1 (sPD-L1) play a significant role in immunologic suppression.

Objectives. To explore expression of SPD-1 and SPD-L1 in a penetrative corneal transplantation model and its relationship with transplant rejection.

Material and methods. Autologous corneal transplantation rat models and allogeneic corneal transplantation rat models were used as the control group and the experimental group, respectively. Changes of the transplanted grafts were observed under a slit-lamp microscope. Hematoxylin-eosin (H\&E) staining was applied to examine the histopathological features of the corneal grafts. Flow cytometry was used to analyze $\mathrm{CD}^{+} \mathrm{CD}^{2} 5^{+}$Treg in the serum and spleen. The sPD-1, SPD-L1, interleukin 10 (IL-10) and interleukin 4 (LL-4) levels in serum and the aqueous humor of the rats were detected using enzyme-linked immunosorbent assay (ELISA).

Results. After the operation, no transplant rejection occurred in the control group. Flow cytometry results showed that expressions of $\mathrm{CD} 4^{+} \mathrm{CD} 25^{+}$Treg in serum in the experimental group were lower than those in the control group $(p<0.05)$. The ELISA results showed that after the operation, SPD-1 and SPD-L1 expression levels in serum in the experimental group were higher than in the control group (all $p<0.05$ ). After the operation, LL-10 and IL-4 content in serum in the experimental group was lower than in the control group (all $p<0.05$ ). The SPD-1/sPD-L1 ratio in the experimental group was higher than in the control group.

Conclusions. Increases of SPD- 1 content and decreases of $C D 4^{+} C D 25^{+}$Treg, IL-10 and IL-4 levels may be involved in corneal allograft rejection. Dynamic detection of the content of SPD-1 and SPD-L1 in serum and aqueous humor after the operation would help in understanding the local immune response in a clinical setting and predicting the occurrence of corneal graft rejection.

Key words: SPD-1, SPD-L1, CD4+CD25+Treg, corneal transplantation, immunological rejection 


\section{Introduction}

Various ocular surface diseases and traumatism can cause irreversible corneal blindness, and corneal transplantation is the most important way of restoring sight. Although the success rate of transplantation operations is high, immunological rejection is still a leading cause of corneal transplantation failure. ${ }^{1,2}$ Immunosuppressors are used in clinical practice and have shown remarkable results. However, the high price and obvious toxicity of immunosuppressors, as well as their side effects, limit the long-term use of immunosuppressors. Hence, in recent years, more attention has been paid to how to suppress corneal transplant rejection and prolong the survival time of corneal grafts by inducing transplant graft tolerance. Much evidence has shown that activating the T lymphocyte requires co-stimulatory signals, which can help successfully induce transplant graft tolerance in transplantation immunity. ${ }^{3,4}$ Programmed death protein-1 (PD-1) and programmed death ligand-1 (PD-L1) are important negative pathways for regulating the T lymphocyte. . $^{5,6}$

$\mathrm{CD} 4^{+} \mathrm{CD} 25^{+}$Treg has immunosuppressive effects, plays a crucial role in suppressing the immune response, and can enhance immune tolerance by suppressing responding T cells. ${ }^{7,8}$ Research has shown that the PD-1/PD-L1 pathway plays an important part in balancing $\mathrm{CD} 4{ }^{+} \mathrm{CD} 25^{+}$Treg autoimmunity and negatively regulating transplant rejection. ${ }^{9}$ Programmed death protein-1 and PD-L1 exist in membrane and soluble forms, namely soluble programmed death protein-1 (sPD-1) and soluble programmed death ligand-1 (sPD-L1) ${ }^{10}$ Programmed death ligand-1 is a significant factor for corneal immune-privileged status, which can prevent the occurrence of transplant rejection. ${ }^{11}$ In addition, it has been reported that excessive SPD-1 leads to immunologic injury by blocking the PD-1/PD-L1 pathway. ${ }^{12}$ In other words, expression of SPD-1 may increase corneal transplant rejection by restraining the PD-1/PD-L1 signal. Some studies have indicated that the $\mathrm{CD} 4{ }^{+} \mathrm{CD} 25^{+}$Treg cell is related to successful allotransplantation and expression of Foxp3 on the CD4 ${ }^{+} \mathrm{CD} 25^{+}$Treg cell is one of the vital factors for corneal allograft survival. ${ }^{13,14}$ However, research on the functions and relationships of sPD-1/ sPD-L1, CD $4{ }^{+} \mathrm{CD} 25^{+}$Treg and correlating factors in corneal transplantation immunity is still rare. This research would establish penetrative corneal transplantation models in rats and primarily explore their functions and relations in transplant rejection after corneal transplantation.

\section{Material and methods}

\section{Experimental animals}

Thirty healthy female Sprague Dawley (SD) rats were used as donors and 70 female Wistar rats as receptors. All rats were clean, 6-8-weeks old, weighed 180-220 g, and were provided by the Animal Experiment Center of Southern Medical University (Kanton, China)

The rats were divided into 2 groups. The control group, used $60 \mathrm{Wistar}$ rats as donors and receptors to conduct autologous corneal transplantation. In the experimental group allogeneic corneal transplantations were performed that included 30 SD donor rats and 60 receptor Wistar rats. Another $10 \mathrm{Wistar}$ rats were used to detect preoperative values. This research was examined and approved by the Southern Medical University Ethics Committee. All procedures were performed and animals were cared for and treated in accordance with the policies of the Association for Research in Vision and Ophthalmology Department for the Use of Animals in Ophthalmic and Vision Research.

\section{Reagents}

Fluorescent monoclonal antibodies Anti-Rat CD25 perCP-eFluor ${ }^{\circledR} 710$ and Anti-Rat CD4 FITC were bought from Affymetrix eBioscience (Thermo Fisher Scientific, Waltham, USA). Enzyme-linked immunosorbent assay (ELISA) kits for interleukin 10 (IL-10), interleukin 4 (IL-4), PD-1, and PD-L1 were bought from Sango Biotech (Shanghai, China).

\section{Experimental methods and steps}

\section{Establishing allogeneic corneal transplantation models}

According to the method described by Williams et al., binoculus corneas were taken from all donor rats; receptor rats were operated on their right eyes. ${ }^{15}$ Donors and receptors were injected with $10 \%$ chloral hydrate $(35-42 \mathrm{mg} / \mathrm{kg})$ intraperitoneal injection of anesthesia and received 5\% hydrochloric acid, proparacaine eye drops and compound tropicamide eye drops 3 times each 15 min before the operation. The whole operation was conducted aseptically with sterile drapes under a microscope. First, the corneal grafts of donor SD rats were taken with a $3.3-\mathrm{mm}$ trephine and placed into a Petri dish under the protection of viscoelastic sodium hyaluronate. A right-eye implant bed for receptors was made with a 3.0-mm trephine. Viscoelastic sodium hyaluronate was injected during the operation to maintain anterior chamber depth. Grafts were placed on the implant bed and 8 interrupted stitches were sutured with a 10-0 nylon suture without embedding thread residue. After the operation, a proper amount of balanced salt solution was injected into the anterior chamber and levofloxacin eye ointment was applied in the conjunctival sac. The eyelid was sutured with a 5-0 silk thread and the stitches were removed 1 day after the operation.

\section{Establishing autologous corneal transplantation models}

Corneal grafts of the Wistar rats were taken with a 3.0$\mathrm{mm}$ trephine and rotated about $180^{\circ}$. Eight interrupted stitches were sutured with a 10-0 nylon suture in situ. 
The following steps were the same with allogeneic corneal transplantation models.

\section{Observing immunological rejection \\ after corneal transplantation}

The corneal grafts in each group were observed under a slit-lamp microscope and records were made of the transparency, angiogenesis growth condition and rejection response, starting 1 day after the operation. The observation period was 28 days, once a day. Once such ocular complications as graft infection, hyphema, loss of anterior chamber, and anterior synechia occurred because of the operation and other factors, the rat would be removed and replaced. According to the scoring criteria of Larkin et al., ${ }^{16}$ scores would be given considering the opacification, edema and angiogenesis of the corneal grafts. Rejection index (RI) was calculated by adding up the scores of all 3 indexes. The rejection exits were defined when RI $>5$ or the opacification of the corneal graft score reached 3 .

\section{Histopathological examination}

Two receptor rats in each group were killed 3 days, 7 days, 14 days, 4 weeks, and 8 weeks after the operation, respectively. The whole cornea was cut off from the selflimbal grafts and the corneal tissue was fixed in $10 \%$ paraformaldehyde solution to receive conventional dehydrating and paraffin embedding. Hematoxylin-eosin (HE) staining and optical microscopy were used in the histopathological examination.

\section{Flow cytometry detection}

Four receptor rats in each group were killed 3 days, 7 days, 14 days, 4 weeks, and 8 weeks after the operation, respectively. Anti-clotting serum and spleen cell suspension were detected in sterile conditions with flow cytometry. Anterior chamber aqueous humor was taken for the ELISA detection.

\section{Enzyme-linked immunosorbent assay}

Six receptor rats in each group were killed 3 days, 7 days, 14 days, 4 weeks, and 8 weeks after the operation, respectively. Serum and anterior chamber aqueous humor were taken in sterile conditions. The SPD-1, sPD-L1, IL-10, and IL-4 concentration levels were detected in serum and anterior chamber aqueous humor.

\section{Statistical methods}

The experimental data was expressed as $\mathrm{x} \pm \mathrm{s}$ and analyzed with SPSS v. 20.0 software (IBM Corp., Armonk, USA). Survival rates of the corneal grafts were analyzed with Kaplan-Meier survival curves. The independent samples t-test was used to compare the detection results in both groups.
A nonparametric rank-sum test was used when non-normal distributions of unequal variances occurred. The data was considered statistical significant when $\mathrm{p}<0.05$.

\section{Results}

\section{Postoperative slit-lamp microscope observation and comparison of graft survival time}

The comparison of graft survival time is shown in Fig. 1. In the experimental group, 3 days after the operation, there was a slight edema and opacity on the cornea and limbus cornea vessels dilated, while the cornea obviously thickened, and hydrops and opacity increased, with new blood vessels sprouting on day 7 . Fourteen days after the operation, corneal graft edema worsened while the grafts thickened and bulged outwards. In addition, opacity of the cornea became apparent with the iris blurred, and new blood corneal vessels developed and embedded into the grafts. Four weeks after the operation, corneal graft edema lightened; grafts thickened and bulged outwards; corneas stayed turbid; new blood corneal vessels grew near the center. Eight weeks after the operation, corneal grafts showed little edema and were overgrown by new blood vessels; pupils could be indistinctly detected; stitches loosened and came off to different extents (Fig. 1A).

In the control group, 3 days after the operation, there was a situation similar to that in the experimental groups and the iris was visible. After 7 days, there was a slight edema in corneal grafts while the iris was visible and the limbus cornea vessels were dilated. After 14 days, corneal graft edema disappeared and the iris vessels were visible. Four weeks after the operation, there was no apparent edema, thickening and opacity of the corneal grafts, and a small number of new blood vessels occurred around the stitches. Eight weeks after the operation, corneal grafts and the iris could be observed. Additionally, a small number of new blood vessels formed around the stitches (Fig. 1B). The survival rates of the grafts are shown in Fig. 2. After the operation, the corneal graft survival rate in the control group remain stable. Unlike the control group, the average survival time between the corneal grafts in the experimental group was $13.1 \pm 2.2$ days. The difference between the corneal graft survival curves was statistically significant (ts $=21.026, \mathrm{p}=0.000)$.

\section{Histopathological examination}

The histopathological features were different between the experimental group and the control group after the operation (Fig. 3). In the experimental group, after the operation, the stroma collagen fiber structure of the corneal grafts was arranged chaotically and proliferated. In addition, not only the new blood vessels appeared over the corneal 
A

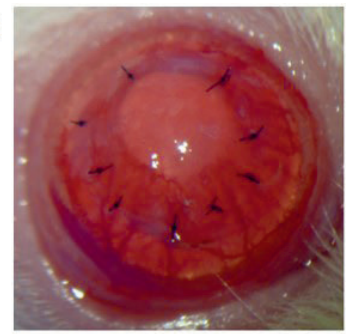

B

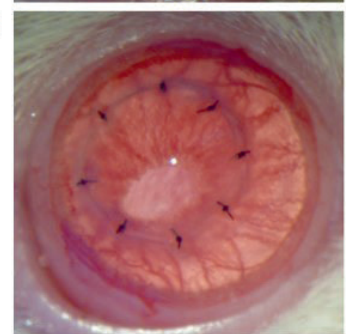

3 days
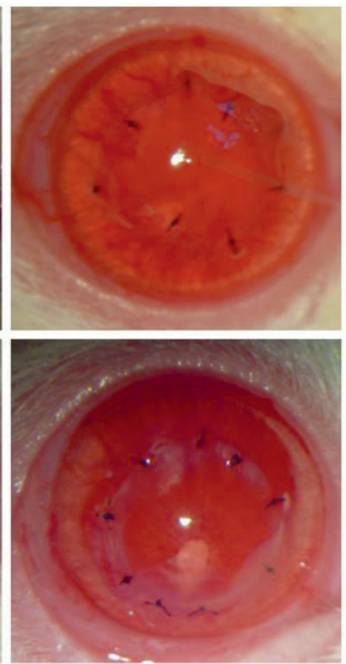

7 days
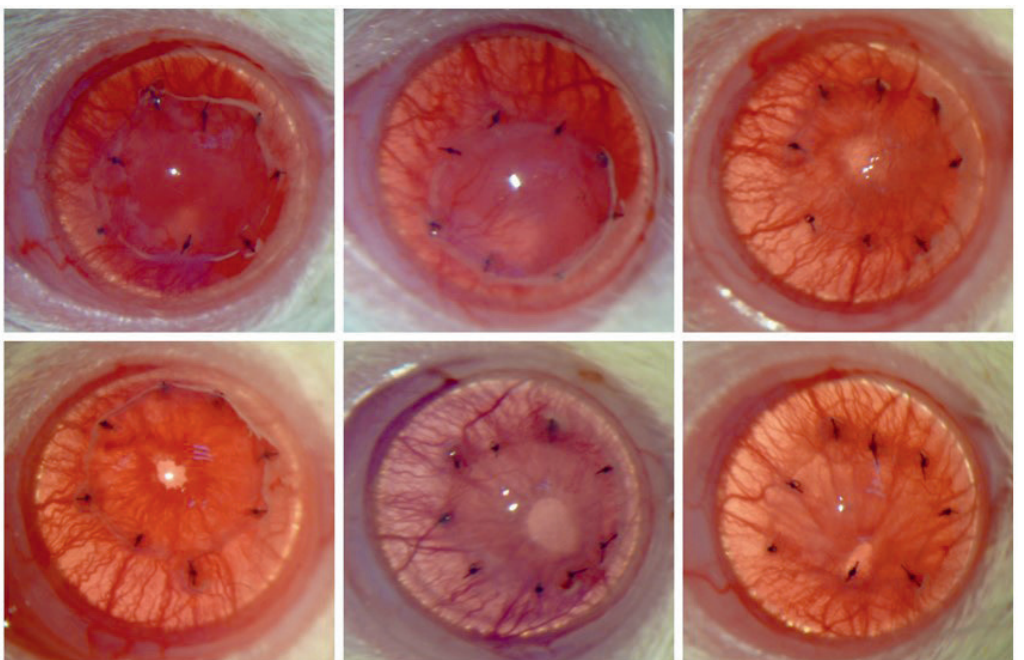

14 days

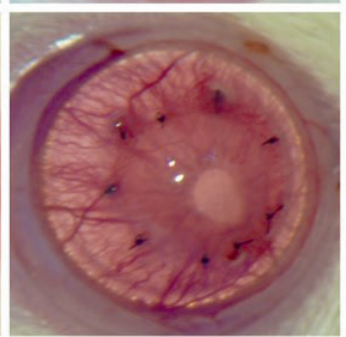

4 weeks

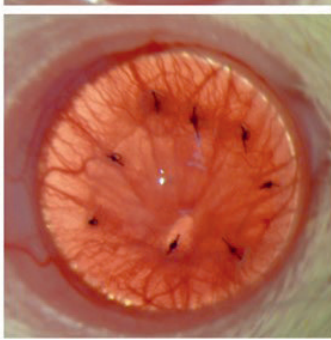

8 weeks

Fig. 1. Postoperative slit-lamp observation results. A. The results showed the change of cornea and limbus cornea vessels in the experimental group within 3 days, 7 days, 14 days, 4 weeks, and 8 weeks after the operation. The corneal graft has slight edema in 3 days after the operation and new blood vessels sprouted on the $7^{\text {th }}$ day. Edema worsened in 14 days and in 8 weeks edema was reduced; B. After the operation, in the control group, corneal graft edema disappeared within 14 days after the operation. There were few new blood vessels generated. The difference of corneal graft survival curves is statistically significant (ts = 21.026, $p=0.000)$.

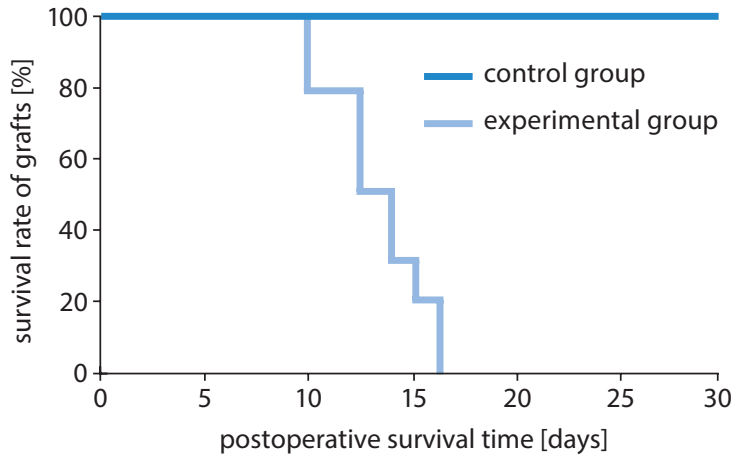

Fig. 2. The results show the comparison of survival rates of the corneal graft between the control group and experimental group

The survival rate of the corneal graft in the experimental group decreased as time increased. The survival rate of the corneal graft in the control group remained unchanged

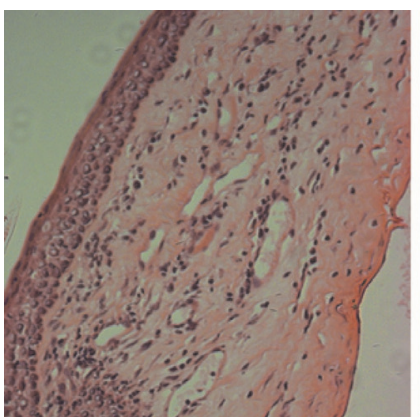

experimental group

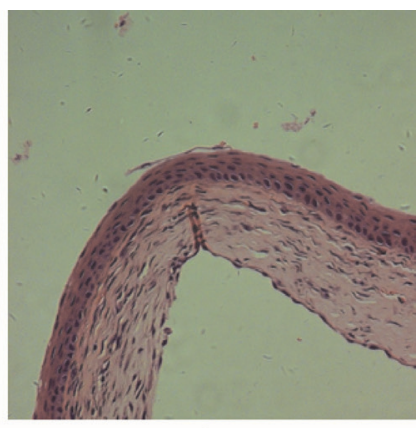

control group
Fig. 3. Comparison of histopathological features between the experimental group and control group

After the operation, the stroma collagen fiber structure of the corneal grafts ranked irregularly in the experimental group while it was arranged orderly in the control group. New blood vessels, spongiform and disintegrating can be observed in the experimental group. There was no significant change in the control group.

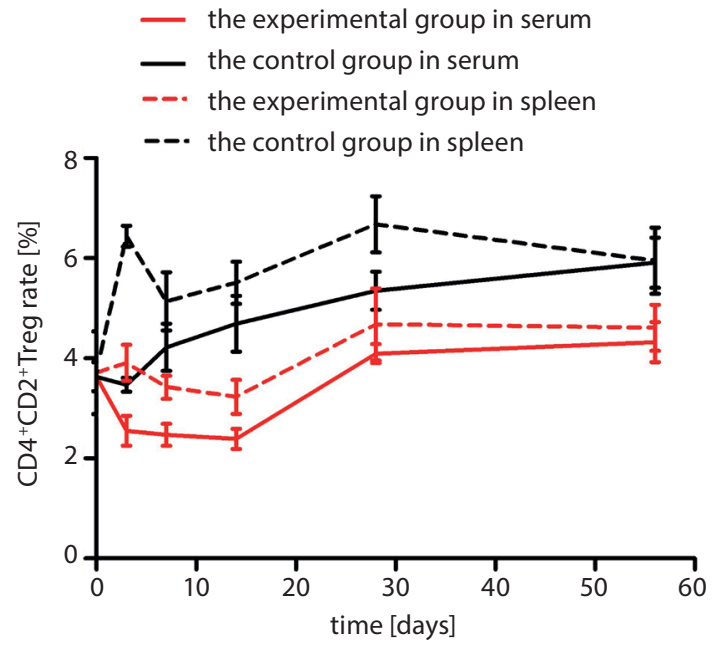

Fig. 4. Expression level of $C D 4^{+} \mathrm{CD} 25^{+}$Treg in serum and spleen

The $\mathrm{CD} 4^{+} \mathrm{CD} 25^{+}$Treg cell expression levels in serum and the spleen in the experimental group were all higher than in the control group (all $p<0.05$ ).

periphery, but also spongiform and disintegrating appeared on the epithelial grafts. Additionally, a lot of inflammatory cells were infiltrated after the operation. However, compared to the experimental group, no obvious thickening of the corneal grafts occurred in the control group. Additionally, the stroma collagen fiber structure was basically arranged orderly. Only a small amount of inflammatory cells was infiltrated. No obvious new blood vessels had formed.

\section{Detection results of flow cytometry}

The expression level of $\mathrm{CD} 4^{+} \mathrm{CD} 25^{+}$Treg in the serum and spleen is shown in Fig. 4. The expression level of $\mathrm{CD} 4^{+} \mathrm{CD} 25^{+}$Treg in serum in the control group decreased 
to its lowest point at day 3 and began increasing gradually. It exceeded the preoperative value after 7 days and reached a peak 8 weeks after the operation. In the experimental group, the expression level of CD $4{ }^{+} \mathrm{CD} 25^{+}$Treg in serum started to fall 3 days after the operation, dropping below its preoperative value. It declined to its lowest level after 14 days and then gradually increased to a peak 8 weeks after the operation. $\mathrm{CD} 4{ }^{+} \mathrm{CD} 25^{+}$Treg content in serum in the experimental group was higher than in the control group at each time point after the operation. The group difference was statistically significant $(t=5.564,6.722,7.667,5.869,4.954$; $\mathrm{p}=0.001,0.001,0.000,0.001,0.003)$. In both groups, the expression level of $\mathrm{CD} 4{ }^{+} \mathrm{CD} 25^{+}$Treg in the spleen immediately increased 3 days after the operation. In the control group, it gradually rose from day 14 and reached a peak 4 weeks after the operation. The expression level of $\mathrm{CD} 4{ }^{+} \mathrm{CD} 25^{+}$Treg was higher than the preoperative value. In the experimental group, the expression level of CD $4{ }^{+} \mathrm{CD} 25^{+}$Treg started to decrease after 7 days and reached its lowest point 14 days after the operation. The expression level of $\mathrm{CD} 4^{+} \mathrm{CD} 25^{+}$Treg in the spleen in the experimental group was prominently lower than in the control group. The group difference was statistically significant $(\mathrm{t}=11.885,5.461,8.469,4.407,3.323$; $\mathrm{p}=0.000,0.002,0.000,0.005,0.016)$.

\section{Expression level of sPD-1}

In the experimental group, the expression level of sPD-1 in serum started to increase 3 days after the operation, reached its highest point after 14 days and gradually decreased to its lowest point, reaching 8 weeks after the operation. The sPD-1 content was higher than the preoperative value at each time point after the operation. In the control group, the expression level of sPD-1 in serum was lower than the preoperative value at each time point, except on the $3^{\text {rd }}$ day after the operation, and reached its lowest point 8 weeks after the operation. The expression level of sPD-1 in serum in the experimental group was prominently higher than in the control group. The group difference was statistically significant $(\mathrm{t}=2.399,3.013,4.574,2.921,2.817$; $\mathrm{p}=0.037,0.013,0.001,0.015,0.018)$. Similarly, the expression level of sPD-1 in the experimental group in aqueous humor immediately increased after 3 days, reached a peak 14 days after the operation and started to decrease afterwards. The expression level of sPD-1 was higher than the preoperative value. In the control group, it reached a peak 3 days after the operation and gradually decreased. The expression level of sPD-1 in the experimental group was prominently higher than in the control group at each time point after the operation. The group difference was statistically significant $(t=2.409,2.964,4.128,2.375$, 2.403; $\mathrm{p}=0.037,0.014,0.002,0.039,0.037)$ (Fig. 5A).

\section{Expression level of sPD-L1}

In both groups, the expression level of SPD-L1 in serum decreased 3 days after the operation, while it began to increase gradually in the control group. In the experimental group, it was gradually reduced to its lowest point, reaching it 14 days after the operation, and then slightly increased. The expression level of sPD-L1 in serum in the experimental group was lower than in the control group at each time point after the operation. Only the group difference 14 days after the operation was statistically significant. In the control group, the expression level of sPD-L1 in aqueous humor immediately decreased on the $3^{\text {rd }}$ day after the operation and gradually increased afterwards. In the experimental group, the expression level of sPD-L1 began to decrease on the $3^{\text {rd }}$ day, reached its lowest point 14 days after the operation and then started to increase gradually. The expression level of sPD-L1 in aqueous humor in the experimental group was lower than in the control group at each time point after the operation; however, the group difference was not statistically significant (Fig. 5B).

\section{Expression level of interleukin 10}

In both groups, IL-10 content in serum decreased to its lowest point 14 days after the operation and gradually increased afterwards. The expression level of IL-10 in serum in the experimental group was prominently lower than in the control group. The group difference was statistically significant $(\mathrm{t}=4.515,4.663,3.591,2.673,3.940 ; \mathrm{p}=0.001,0.001,0.005$, $0.023,0.003)$. In the control group, the expression level of IL-10 in aqueous humor gradually increased to a peak, which was reached 4 weeks after the operation. In the experimental group, the expression level of IL-10 in aqueous humor started to decrease 3 days after the operation, reaching its lowest point 14 days after the operation, which was lower than the preoperative value. The expression level of IL-10 in aqueous humor in the experimental group was prominently lower than in the control group. The group difference was statistically significant $(t=3.609,5.451,6.868,5.424$, 5.432; $\mathrm{p}=0.005,0.000,0.000,0.000,0.000)($ Fig. 5C).

\section{Expression level of interleukin 4}

In both groups, IL-4 content in serum decreased 3 days after the operation. In the control group, it gradually increased 7 days after the operation and only the expression level of IL- 4 after 3 days was higher than the preoperative value. The expression level of IL-4 in serum in the experimental group was prominently lower than that in the control group. The group difference was statistically significant $(\mathrm{t}=2.338,2.644,4.561,4.896,6.882 ; \mathrm{p}=0.042$, $0.025,0.001,0.001,0.000)$. Similarly, IL- 4 content in both groups in aqueous humor decreased 3 days after the operation. In the experimental group, it sharply decreased and reached its lowest point 14 days after the operation. In the control group, it gradually increased from the $7^{\text {th }}$ day after the operation. Interleukin 4 content in aqueous humor in the experimental group was prominently lower than in the control group. The group difference was statistically significant $(t=7.323,10.639,11.190,11.860,9.327$; $\mathrm{p}=0.000,0.000,0.000,0.000,0.000)$ (Fig. 5D). 

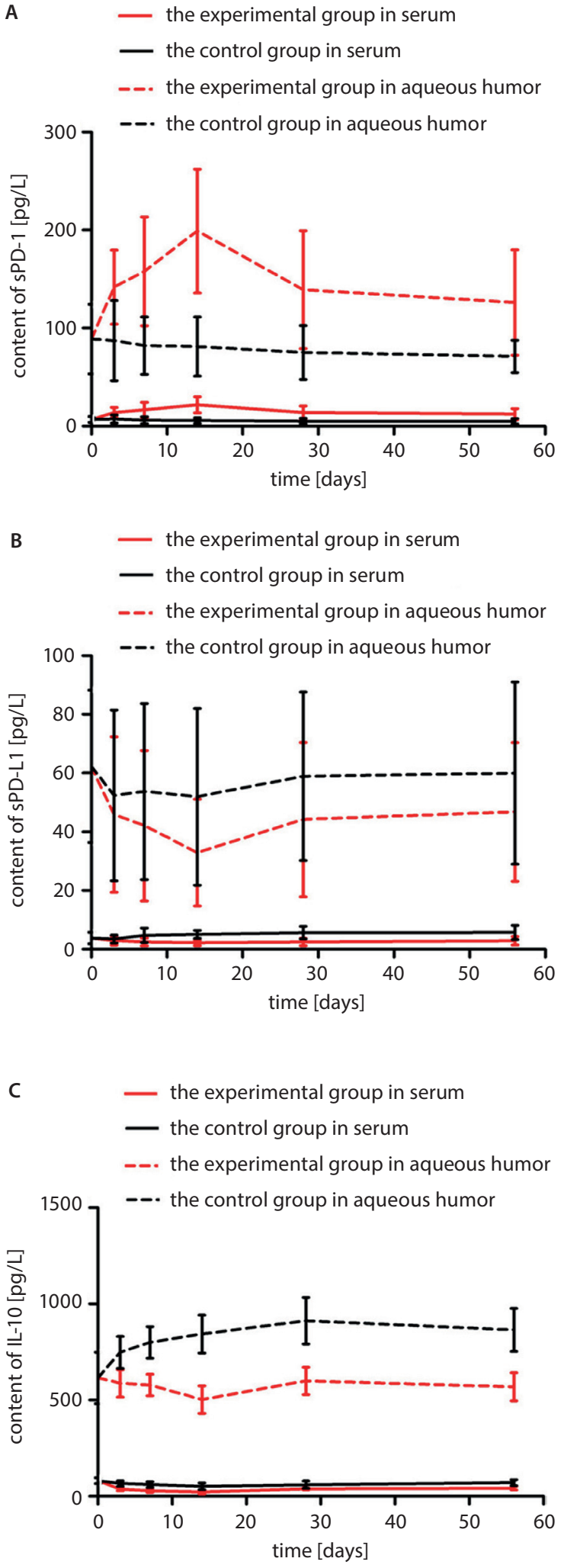

\section{Analysis of ratios between SPD-1 and sPD-L1 in control and experimental groups}

According to the ELISA results, the expression level of sPD-1 in the experimental group was prominently higher than in the control group while the expression level of sPD-L1 in the experimental group was lower
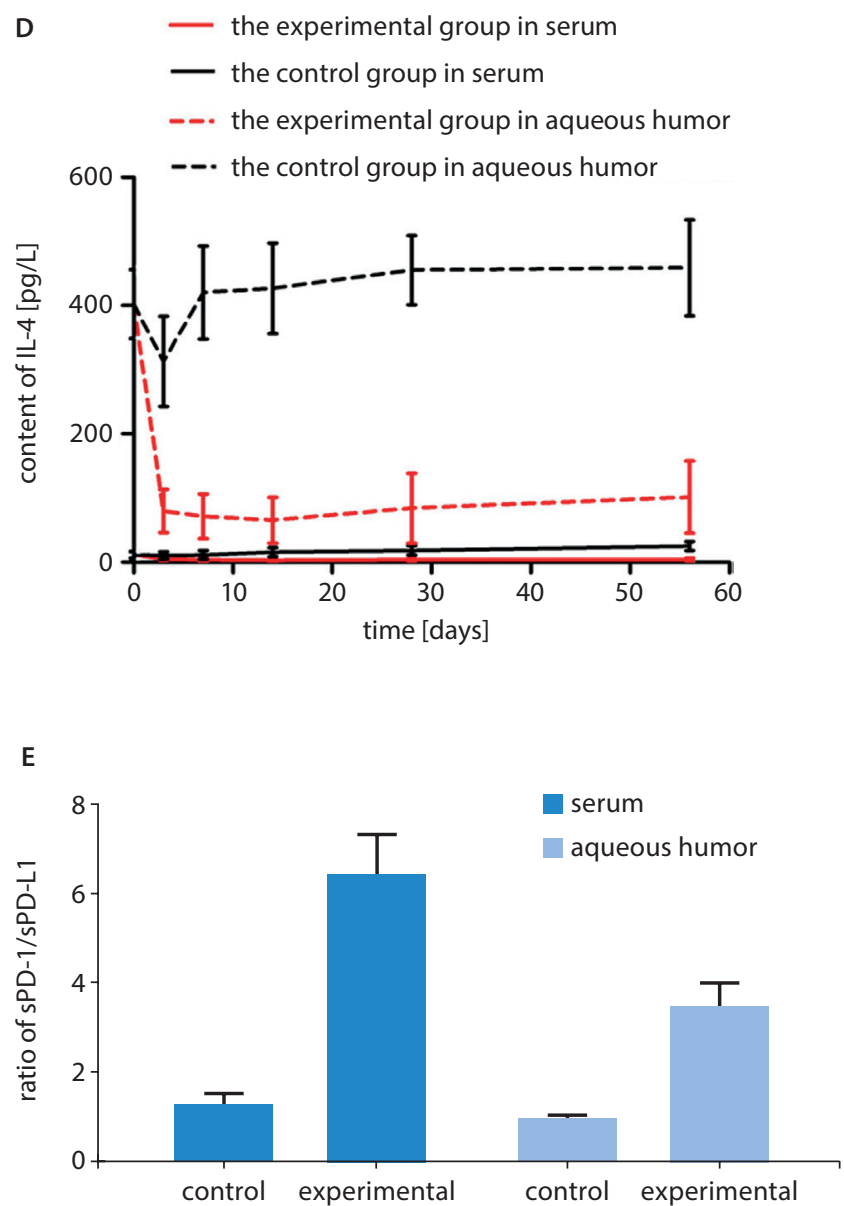

Fig. 5. Expression level of soluble programmed death protein-1 (sPD-1), soluble programmed death ligand protein-1 (sPD-L1), interleukin 10 (IL-10), interleukin 4 (IL-4), and ratios analyzed between SPD-1 and SPD-L1 in serum and aqueous humor

A. The content of sPD-1 in aqueous humor in the experimental group was higher than in the control group (all $p<0.05$ ). The expression level of serum SPD-1 in the experimental group was higher than in the control group (all $p<0.05$ ); B. In aqueous humor, the content of SPD-L1 in the control group was higher than in the experimental group (all $p<0.05$ ). There was no significant difference of the serum SPD-L1 content between the experimental and control groups; $\mathrm{C}$. The expression level of IL-10 in aqueous humor in the control group was lower than in the experimental group (all $p<0.05$ ). The expression level of serum IL-10 in the control group was higher than in the experimental group (all $p<0.05$ ); D. The expression level of IL-4 in aqueous humor in the control group was higher than in the experimental group (all $p<0.05$ ). The expression level of serum IL-4 in the control group was higher than in the experimental group (all $p<0.05$ ); E. Both ratios of serum SPD-1/SPD-L1 and aqueous humor in the experimental group were higher than in the control group.

than in the control group. After analysis, it was found that the $\mathrm{SPD}-1 / \mathrm{sPD}-\mathrm{L} 1$ ratio in serum in the experimental group was much higher than in the control group. The sPD-1/sPD-L1 ratios in serum in the control group and experimental group were $1.259 \pm 0.499$ and $6.319 \pm 2.317$, respectively, and the difference was statistically significant $(\mathrm{p}<0.05)$. Similarly, the ratio of sPD-1/ sPD-L1 in aqueous humor in the experimental group was higher than in the control group. The sPD-1/sPD-L1 ratios in aqueous humor in the control group and experimental 
group were $1.443 \pm 0.204$ and $3.749 \pm 1.341$, respectively, and the difference was statistically significant $(\mathrm{p}<0.05)$ (Fig. 5E).

\section{Discussion}

Transplant rejection is a major cause of corneal transplantation failure. Therefore, the core issue of corneal transplantation is how to inhibit transplant rejection. At present, prevention and treatment of transplant rejection after corneal transplantation mainly focuses on the effect phase using eye local and systemic immune inhibitors, angiogenesis inhibitors, etc., ${ }^{17-22}$ but these drugs can disturb or destroy the normal immune function and environment. Furthermore, the concentration of the drugs will eventually decrease below effective concentration after body metabolism, and transplant rejection may still happen. Thus, if the proliferation and differentiation of immune effector cells can be restrained and prevented from entering the effect phase, the effect of inducing and transplanting immunological tolerance will be achieved, which can effectively and enduringly inhibit transplant rejection and prolong survival time of the grafts. This is one important direction of future study on transplantation immunological tolerance.

In recent years, the co-stimulating signal has become an important issue in immunological research. In transplantation immunity, activating the $\mathrm{T}$ lymphocyte needs co-stimulating signals to successfully induce transplantation immunological tolerance. Currently known co-stimulating molecules include the B7 family (immunoglobulin superfamily), tumor necrosis factor (TNF) family and cytokine family negative co-stimulating molecules, while the PD-1/PD-L1 pathway is an important negative regulating pathway of $\mathrm{T}$ lymphocytes. ${ }^{5-7,23-25}$ Research shows that in the PD-1/PD-L1 pathway, PD-1 works as an immune inhibitory receptor and is mainly expressed in activated $\mathrm{T}$ cells. ${ }^{26-28}$ By interacting with PD-L1, PD-1 can promote immunological suppression and regulation, which can help induce and maintain immunological tolerance. ${ }^{29-32}$ Additionally, studies show that $\mathrm{CD} 4{ }^{+} \mathrm{CD} 25^{+}$Treg has features of immune incompetence and inhibition, which play a key role of effector $T$ cells in maintaining the body's immune response steady state and the immunological suppression function of immunological tolerance after induction organ transplantation. ${ }^{33,34}$ It was first confirmed by Hall et al. that CD $4{ }^{+} \mathrm{CD} 25^{+}$Treg could induce transplantation immunological tolerance. ${ }^{35}$ $\mathrm{CD} 4{ }^{+} \mathrm{CD} 25^{+}$Treg can also secrete cytokines such as IL-10 and $1 \mathrm{~L}-4$ to suppress the immune response. ${ }^{36-38}$ In this research, the content of serum and aqueous humor increased while the expression level of $\mathrm{CD} 4{ }^{+} \mathrm{CD} 25^{+}$Treg in serum and the spleen decreased after the operation in the experimental group. In the control group, sPD-1 content in serum and aqueous humor decreased and the expression level of $C D 4+C D 25^{+}$Treg in serum and the spleen increased. In the experimental group, sPD-1 content was significantly higher than the preoperative value at the beginning of rejection while the expression level of $\mathrm{CD} 4{ }^{+} \mathrm{CD} 25^{+}$Treg was markedly lower than the preoperative value, indicating that SPD- 1 and $C D 4{ }^{+} \mathrm{CD} 25^{+}$Treg were related to transplant rejection after corneal transplantation.

In addition, it is worth noting that the rising expression level of sPD-1 was opposite to the increasing tendency of IL-10 and IL-4 content in serum cytokines and aqueous humor. The IL-10 and IL-4 content in aqueous humor were higher than that in serum and the change range was broader. Therefore, we can speculate on the basis of these results that when transplant rejection happens after corneal transplantation, the expression level of sPD-1 will increase. It may be combined with PD-L1 to inhibit the combination of membrane-type PD-1 and PD-L1, and prevent PD-1/PD-L1 from conveying negative stimulation signals after combination, thus inhibiting the function of $\mathrm{CD} 4{ }^{+} \mathrm{CD} 25^{+}$Treg and indisposing it to conduct immunoregulation. Meanwhile, the decrease of IL-10 and IL-4 secretion may weaken transplant rejection inhibition after corneal transplantation. The group difference of the postoperative expression level of SPD-L1 in serum and aqueous humor is not statistically significant. The changing tendency does not have a clear relationship between transplant rejection and $\mathrm{CD} 4{ }^{+} \mathrm{CD} 25^{+}$Treg and no regularity was found. Yet in the analysis of sPD-1/sPD-L1 ratios of the 2 groups, it was found that at the same time point, the mean sPD-1/ SPD-L1 ratio in serum and aqueous humor in the experimental group was greater than in the control group and it reached its highest level 14 days after the operation. This result showed that the abnormal expression of sPD-1/ SPD-L1 may have certain relevance to transplant rejection after corneal transplantation. The content ratio of sPD-1 and SPD-L1 in serum and aqueous humor can work as an important reference index of transplant rejection after corneal transplantation. Monitoring the concentration changes of sPD-1 and sPD-L1 can help to predict and diagnose the occurrence of rejection at an early stage.

\section{Conclusions}

To sum up, the experiment preliminarily proved that in transplant rejection after rat corneal transplantation, increaseD expression level of sPD-1 may inhibit the combination of membrane-type PD-1 and PD-L1, blocking the negative adjustment of PD-1/PD-L1 on $\mathrm{CD} 4^{+} \mathrm{CD} 25^{+}$Treg after the combination and impeding normal immunological tolerance. The decreasing content of IL-10 and IL-4 secreted in serum and aqueous humor may weaken the protection of the body's immune suppression and increase immune rejection, as well as influence the survival time of corneal grafts. 
Two problems are still unclear: whether sPD-1/sPD-L1 work as collaborative or inhibiting regulating factors of PD-1/PD-L1; and the specific mechanism of rejection after corneal transplantation. These issues should be studied further.

\section{References}

1. Niederkorn JY. The immune privilege of corneal grafts. J Leukoc Biol. 2003;74(2):167-171.

2. Price MO, Thompson RW Jr, Price FW Jr. Risk factors for various causes of failure in initial corneal grafts. Arch Ophthalmol. 2003;121(8): 1087-1092.

3. Nurieva R, Thomas $S$, Nguyen $T$, et al. T-cell tolerance or function is determined by combinatorial costimulatory signals. EMBO J.2006; 25(11):2623-2633.

4. Snanoudj R, de Preneuf $\mathrm{H}$, Creput $\mathrm{C}$, et al. Costimulation blockade and its possible future use in clinical transplantation. Transpl Int. 2006;19(9):693-704.

5. Cai G, Karni A, Oliveira EM, Weiner HL, Hafler DA, Freeman GJ. PD-1 ligands, negative regulators for activation of naive, memory and recently activated human $\mathrm{CD}^{+} \mathrm{T}$ cells. Cell Immunol. 2004; 230(2):89-98.

6. Latchman YE, Liang SC, Wu Y, et al. PD-L1-deficient mice show that PD-L1 on T cells, antigen-presenting cells, and host tissues negatively regulates T cells. Proc Natl Acad Sci U S A. 2004;101(29):10691-10696.

7. Taams LS, Vukmanovic-Stejic M, Smith J, et al. Antigen-specific T cell suppression by human $\mathrm{CD}^{+}{ }^{+} \mathrm{CD} 25^{+}$regulatory T cells. Eur JImmunol. 2002;32(6):1621-1630.

8. Dieckmann D, Plottner H, Berchtold S, Berger T, Schuler G. Ex vivo isolation and characterization of $\mathrm{CD} 4(+) \mathrm{CD} 25(+) \mathrm{T}$ cells with regulatory properties from human blood. J Exp Med. 2001;193(11):1303-1310.

9. Kitazawa Y, Fujino M, Wang Q, et al. Involvement of the programmed death-1/programmed death-1 ligand pathway in $\mathrm{CD} 4^{+} \mathrm{CD} 25^{+}$regulatory T-cell activity to suppress alloimmune responses. Transplantation. 2007;83(6):774-782.

10. Zhang X, Schwartz JC, Guo X, et al. Structural and functional analysis of the costimulatory receptor programmed death-1. Immunity. 2004;20(3):337-347.

11. Shen L, Jin Y, Freeman GJ, Sharpe AH, Dana MR. The function of donor versus recipient programmed death-ligand 1 in corneal allograft survival. J Immunol. 2007;179(6):3672-3679.

12. Zhao Y, Jia Y, Li C, Fang Y, Shao R. The risk stratification and prognostic evaluation of soluble programmed death-1 on patients with sepsis in emergency department. Am J Emerg Med. 2018;36(1):43-48.

13. Guo X, Jie Y, Ren D, et al. In vitro-expanded CD4(+)CD25(high) Foxp3(+) regulatory T cells controls corneal allograft rejection. Hum Immunol. 2012;73(11):1061-1067.

14. Cunnusamy K, Paunicka K, Reyes N, Yang W, Chen PW, Niederkorn JY. Two different regulatory $T$ cell populations that promote corneal allograft survival. Invest Ophthalmol Vis Sci. 2010;51(12):6566-6574.

15. Williams KA, Coster DJ. Penetrating corneal transplantation in the inbred rat: A new model. Invest Ophthalmol Vis Sci. 1985;26(1):23-30.

16. Larkin DF, Calder VL, Lightman SL. Identification and characterization of cells infiltrating the graft and aqueous humor in rat corneal allograft rejection. Clin Exp Immunol. 1997;107(2):381-391.
17. Xin M, Wang T, Shi W, Wu X. Experimental efficacy of mycophenolate mofetil implant on high-risk corneal allograft rejection and its biocompatibility in the anterior chamber of rabbits. J Ocul Pharmacol Ther. 2012;28(6):609-617.

18. Fan JC, Chow K, Patel DV, McGhee CN. Corticosteroid-induced intraocular pressure elevation in keratoconus is common following uncomplicated penetrating keratoplasty. Eye (Lond). 2009;23(11):2056-2062.

19. Reinhard T, Mayweg S, Reis A, Sundmacher R. Topical FK506 as immunoprophylaxis after allogeneic penetrating normal-risk keratoplasty: A randomized clinical pilot study. Transpl Int. 2005;18(2):193-197.

20. Belin MW, Bouchard CS, Frantz S, Chmielinska J. Topical cyclosporine in high-risk corneal transplants. Ophthalmology. 1989;96(8): 1144-1150.

21. Stevenson W, Cheng SF, Dastjerdi MH, Ferrari G, Dana R. Corneal neovascularization and the utility of topical VEGF inhibition: Ranibizumab (Lucentis) vs bevacizumab (Avastin). Ocul Surf. 2012;10(2):67-83.

22. Vassileva Pl, Hergeldzhieva TG. Avastin use in high risk corneal transplantation. Graefes Arch Clin Exp Ophthalmol. 2009;247(12):1701-1706.

23. Greenwald RJ, Freeman GJ, Sharpe AH. The B7 family revisited. Annu Rev Immunol. 2005;23:515-548.

24. Watts TH. TNF/TNFR family members in costimulation of T cell responses. Annu Rev Immunol. 2005;23:23-68.

25. Chen L. Co-inhibitory molecules of the B7-CD28 family in the control of T-cell immunity. Nat Rev Immunol. 2004;4(5):336-347.

26. van Dam LS, de Zwart VM, Meyer-Wentrup FA. The role of programmed cell death-1 (PD-1) and its ligands in pediatric cancer. Pediatr Blood Cancer. 2015;62(2):190-197.

27. Pedoeem A, Azoulay-Alfaguter I, Strazza M, Silverman GJ, Mor A. Programmed death-1 pathway in cancer and autoimmunity. Clin Immunol. 2014;153(1):145- 152.

28. Boussiotis VA, Chatterjee P, Li L. Biochemical signaling of PD-1 on T cells and its functional implications. Cancer J. 2014;20(4):265-271.

29. Dai S, Jia R, Zhang X, Fang Q, Huang L. The PD-1/PD-Ls pathway and autoimmune diseases. Cell Immunol. 2014;290(1):72-79.

30. Ott PA, Hodi FS, Robert C. CTLA-4 and PD-1/PD-L1 blockade: New immunotherapeutic modalities with durable clinical benefit in melanoma patients. Clin Cancer Res. 2013;19(19):5300-5309.

31. Gianchecchi E, Delfino DV, Fierabracci A. Recent insights into the role of the PD-1/PD-L1 pathway in immunological tolerance and autoimmunity. Autoimmun Rev. 2013;12(11):1091-1100.

32. Brusa D, Serra S, Coscia, M, et al. The PD-1/PD-L1 axis contributes to T-cell dysfunction in chronic lymphocytic leukemia. Haematologica. 2013;98(6):953-963.

33. Suri-Payer E, Amar AZ, Thornton AM, Shevach EM. CD4 ${ }^{+} C D 25^{+} T$ cells inhibit both the induction and effector function of autoreactive $\mathrm{T}$ cells and represent a unique lineage of immunoregulatory cells. J Immunol. 1998;160(3):1212-1218.

34. Stephens LA, Barclay AN, Mason D. Phenotypic characterization of regulatory $\mathrm{CD}^{+} \mathrm{CD}^{2} 5^{+} \mathrm{T}$ cells in rats. Int Immunol. 2004;16(2):365-375.

35. Hall BM, Pearce NW, Gurley KE, Dorsch SE. Specific unresponsiveness in rats with prolonged cardiac allograft survival after treatment with cyclosporine. III. Further characterization of the $\mathrm{CD}^{+}$suppressor cell and its mechanisms of action. J Exp Med. 1990;171(1):141-157.

36. Schwartz RH. Natural regulatory T cells and self-tolerance. Nat Immunol. 2005;6(4):327-330.

37. Roncarolo MG, Gregori S, Battaglia M, Bacchett, R, Fleischhauer K, Levings MK. Interleukin-10-secreting type 1 regulatory $T$ cells in rodents and humans. Immunol Rev. 2006;212:28-50.

38. Wu K, Bi Y, Sun K, Wang C. IL-10-producing type 1 regulatory T cells and allergy. Cell Mol Immunol. 2007;4(4):269-275. 